\title{
Optimal spectrum sensing in cooperative cognitive two-way relay networks
}

\author{
Ardalan Alizadeh and Seyed Mohammad-Sajad Sadough ${ }^{\text {a) }}$ \\ Cognitive Telecommunication Research Group, \\ Department of Electrical and Computer Engineering, \\ Shahid Beheshti University G. C., Evin 1983963113, Tehran, Iran \\ a)s_sadough@sbu.ac.ir
}

\begin{abstract}
This paper investigates a network consisting of some CR terminals distributed between two primary transceivers. In the proposed network model, CR nodes assist the primary transmission as a two-way amplify-and-forward relaying scheme when the primary transceivers are in operation. By using distributed beamforming techniques among CR nodes, we propose an optimal solution to maximize the performance of spectrum sensing in terms of signal-to-noise ratio (SNR) at the CBS while satisfying the quality of service requirements of primary receivers. We demonstrate the superiority of sensing performance in the proposed method by relaxing the problem into the semidefinite form and we achieve the maximum value of SNR in the CBS by using an iterative algorithm.
\end{abstract}

Keywords: cognitive radio, relaying devices, spectrum sensing hardware

Classification: Wireless circuits and devices

\section{References}

[1] K. B. Letaief and W. Zhang, "Cooperative communications for cognitive radio networks," Proc. IEEE, vol. 97, no. 5, May 2009.

[2] A. B. Gershman, N. D. Sidiropoulos, S. Shahbazpanahi, M. Bengtsson, and B. Ottersten, "Convex optimization-based beamforming," IEEE Signal Processing Magazine, pp. 62-75, May 2010.

[3] M. Grant and S. Boyd, "CVX: Matlab software for disciplined convex programming, version 1.21.," 2010 [Online] http://cvxr.com/cvx

\section{Introduction}

Spectrum sensing is a fundamental part in each cognitive radio (CR) system that avoids harmful interference among primary and secondary users, and to improve the detection reliability, cooperative spectrum sensing involving several secondary users is usually deployed [1]. In order to further increase the spectrum efficiency, the multi-antenna and/or two-way relaying technol- 
ogy can be applied. In this paper, the relay nodes become cognitive users that they can communicate with the cognitive base station (CBS) when the primary transceivers are not in operation. Obviously, this scenario requires a spectrum sensing which is performed at the CBS. We aim at deriving the optimal beamforming weights for the two-way relay network so as to maximize the signal-to-noise ratio (SNR) at the CBS subject to some quality of service (QoS) constraints at the primary transceivers. We will see that our scheme leads to an accurate spectrum sensing at the CBS compared to competitive methods. We show that the investigated beamforming optimization problem can be formulated as a semidefinite form [2]. Then, the semidefinite relaxation (SDR) is applied to change the problem into a convex form and obtain an efficient solution for the convex problem. The so-obtained simplified problem is solved by the available semidefinite programming (SDP) solvers such as CVX [3].

\section{System model}

The system model of the considered system is shown in Fig. 1. In this network model, we have a CR network which consists of $n_{r}$ terminals and one CBS. All of the $\mathrm{CR}$ nodes are located between two primary transceivers. The distance between the two primary nodes is very large and thus they are not able to communicate via a direct link. In this considered scenario, CR nodes are able to sense the presence or absence of primary transceivers and at the same time, they are able to play the relay role for establishing the connection between the primary transceivers. With the assumption that the primary transceivers are set to work as a two-step two-way amplify-and-forward protocol, the received complex signal vector $\mathbf{x}$ of size $n_{r} \times 1$ in CR nodes during the first stage of relaying can be written as:

$$
\mathbf{x}=\sqrt{P_{1}} \mathbf{f}_{1} s_{1}+\sqrt{P_{2}} \mathbf{f}_{2} s_{2}+\boldsymbol{\nu}
$$

where $P_{1}$ and $P_{2}$ are the transmit power of two transceivers and are assumed constant, $s_{1}$ and $s_{2}$ are the information symbols transmitted by transceivers 1 and 2, respectively, $\boldsymbol{\nu}$ is the $n_{r} \times 1$ complex noise vector at the CR terminals,

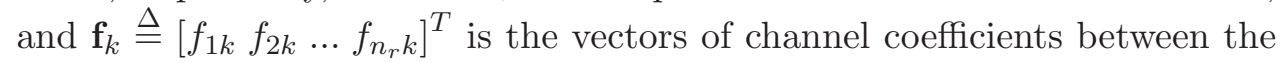
$k$-th $(k=1,2)$ transceiver and the CR terminals, and $(.)^{T}$ is the transpose operator.

In the second step of relaying, each CR node multiplies its received signal by a complex weight $w_{i}^{*}$ and broadcasts it toward the network. Then, the $n_{r} \times 1$ complex vector $\mathbf{t}$ denoting the transmitted signal from the $i$-th $\mathrm{CR}$ terminal is $\mathbf{t}=\mathbf{W} \mathbf{x}$, where the weighting matrix $\mathbf{W}=\operatorname{diag}\left\{\left[w_{1}^{*} w_{2}^{*} \ldots w_{n_{r}}^{*}\right]\right\}$. $\operatorname{diag}\{\mathbf{a}\}$ is a diagonal matrix with diagonal elements equal to the vector $\mathbf{a}$.

\subsection{The SNR at the primary and secondary receivers}

In the second step of relaying, the received signals at the two transceivers $(i=1,2)$ are equal to: 


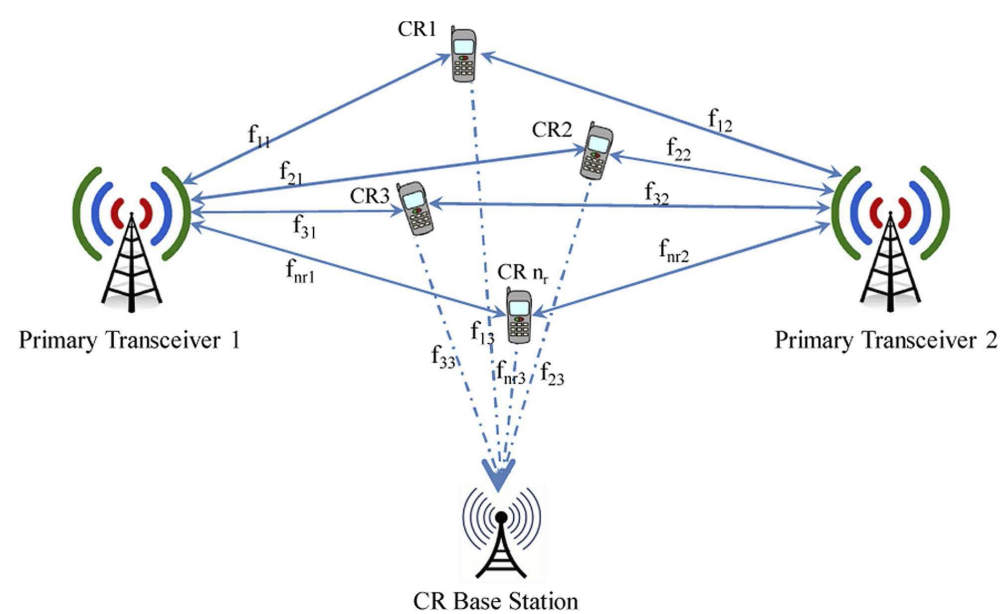

Fig. 1. System model of the considered cognitive two-way relay network.

$$
\begin{aligned}
y_{i} & =\mathbf{f}_{i}^{T} \mathbf{W} \mathbf{x}+n_{i}=\mathbf{f}_{i}^{T} \mathbf{W}\left(\sqrt{P_{1}} \mathbf{f}_{1} s_{1}+\sqrt{P_{2}} \mathbf{f}_{2} s_{2}+\boldsymbol{\nu}\right)+n_{i} \\
& =\sqrt{P_{1}} \mathbf{w}^{H} \mathbf{F}_{i} \mathbf{f}_{1} s_{1}+\sqrt{P_{2}} \mathbf{w}^{H} \mathbf{F}_{i} \mathbf{f}_{2} s_{2}+\mathbf{w}^{H} \mathbf{F}_{i} \boldsymbol{\nu}+n_{i},
\end{aligned}
$$

where $n_{i}$ is the received noise at the $i$-th transceiver during the relaying second step, for $i=1,2, \mathbf{F}_{i} \triangleq \operatorname{diag}\left(\mathbf{f}_{i}\right)$ for $i=1,2, \mathbf{w}=\operatorname{diag}\left\{\mathbf{W}^{H}\right\}$, and $(.)^{H}$ denotes Hermitian transpose. The beamforming weight vector $\mathbf{w}$ is obtained from our optimization problem and can be assumed available at each transceiver. Then, $\sqrt{P_{1}} \mathbf{w}^{H} \mathbf{F}_{1} \mathbf{f}_{1} s_{1}$ is known at primary transceiver 1 and thus the first term in (2) is known at transceiver 1 . Hence, this term can be subtracted from $y_{1}$ and the residual signal can be processed at transceiver 1 to extract the information $s_{2}$ and similarly can be processed at transceiver 2 to extract the information $s_{1}$. Therefore, we define the residual signals $\tilde{y}_{1}=y_{1}-\sqrt{P_{1}} \mathbf{w}^{H} \mathbf{F}_{1} \mathbf{f}_{1} s_{1}$ and $\tilde{y}_{2}=y_{2}-\sqrt{P_{2}} \mathbf{w}^{H} \mathbf{F}_{2} \mathbf{f}_{2} s_{2}$, as the observation signals used at their corresponding transceivers to extract the symbol of the other transceiver.

In the first step, the CBS is only able to make a decision about the presence of a primary signal by sensing the sum of two signals which are received from both of transceivers directly. In the second step of relaying, the CBS have access to the combination of signals received from $n_{r}$ CR nodes. Therefore, the CBS can set the complex weight vector so as to achieve a more correct detection of presence in the beginning of each second step. The received signal $y_{3}$ in the second step of relaying in the CBS is:

$$
\begin{aligned}
y_{3} & =\mathbf{f}_{3}^{T} \mathbf{W} \mathbf{x}+n_{3}=\mathbf{f}_{3}^{T} \mathbf{W}\left(\sqrt{P_{1}} \mathbf{f}_{1} s_{1}+\sqrt{P_{2}} \mathbf{f}_{2} s_{2}+\boldsymbol{\nu}\right)+n_{3} \\
& =\sqrt{P_{1}} \mathbf{w}^{H} \mathbf{F}_{3} \mathbf{f}_{1} s_{1}+\sqrt{P_{2}} \mathbf{w}^{H} \mathbf{F}_{3} \mathbf{f}_{2} s_{2}+\mathbf{w}^{H} \mathbf{F}_{3} \boldsymbol{\nu}+n_{3}
\end{aligned}
$$

where $\mathbf{f}_{3}=\left[\begin{array}{llll}f_{13} & f_{23} & \ldots & f_{n_{r} 3}\end{array}\right]^{T}$ is the vector of channel coefficients between the $\mathrm{CR}$ users and the CBS, and $\mathbf{F}_{3} \triangleq \operatorname{diag}\left(\mathbf{f}_{3}\right)$. The CBS uses the signal $y_{3}$ as a sensing information to make decision about the absence or the presence of the primary signal at the second step of relaying.

We assume a Gaussian distribution for the noise terms with variance $E\left\{\boldsymbol{\nu} \boldsymbol{\nu}^{H}\right\}=\sigma^{2} \mathbf{I}$, and a normalized power for data symbols, i.e., $E\left\{\left|s_{k}\right|^{2}\right\}=$ 
1 , for $k=1,2$, where $E\{$.$\} denotes expectation. Then, the received SNR$ values in the second transmission step at transceivers 1 and 2 can be expressed respectively as:

$$
\mathrm{SNR}_{1}=\frac{P_{2} \mathbf{w}^{H} \mathbf{h} \mathbf{h}^{H} \mathbf{w}}{\sigma^{2}+\sigma^{2} \mathbf{w}^{H} \mathbf{D}_{1} \mathbf{w}}, \text { and } \mathrm{SNR}_{2}=\frac{P_{1} \mathbf{w}^{H} \mathbf{h} \mathbf{h}^{H} \mathbf{w}}{\sigma^{2}+\sigma^{2} \mathbf{w}^{H} \mathbf{D}_{2} \mathbf{w}}
$$

and similarly, the received SNR at the CBS can be expressed as:

$$
\mathrm{SNR}_{3}=\frac{P_{1} \mathbf{w}^{H} \mathbf{h}^{\prime} \mathbf{h}^{\prime H} \mathbf{w}+P_{2} \mathbf{w}^{H} \mathbf{h}^{\prime \prime} \mathbf{h}^{\prime \prime} \mathbf{w}}{\sigma^{2}+\sigma^{2} \mathbf{w}^{H} \mathbf{D}_{3} \mathbf{w}}
$$

where $\mathbf{h} \triangleq \mathbf{F}_{1} \mathbf{f}_{2}=\mathbf{F}_{2} \mathbf{f}_{1}, \mathbf{h}^{\prime} \triangleq \mathbf{F}_{3} \mathbf{f}_{1}, \mathbf{h}^{\prime \prime} \triangleq \mathbf{F}_{3} \mathbf{f}_{2}, \mathbf{D}_{1} \triangleq \mathbf{F}_{1} \mathbf{F}_{1}^{H}, \mathbf{D}_{2} \triangleq \mathbf{F}_{2} \mathbf{F}_{2}^{H}$ and $\mathbf{D}_{3} \triangleq \mathbf{F}_{3} \mathbf{F}_{3}^{H}$. It can be shown that the probability of detection increases with the increase of the SNR at the CBS. Therefore, hereafter, we use $\mathrm{SNR}_{3}$ as the sensing performance criteria.

\section{Optimal spectrum sensing}

In this section, we formulate our optimization problem in order to increase the spectrum sensing performance in the CR network while both transceivers meet their minimum SNR requirements. Thereafter, we change the form of our problem to a convex form and an algorithm is proposed to provide the solution. First, we formulate our optimization problem as:

$$
\max _{\mathbf{w}} S N R_{3} \quad \text { s.t. } \mathrm{SNR}_{1} \geq \gamma_{1}, \quad \mathrm{SNR}_{2} \geq \gamma_{2}, P_{T} \leq P_{T}^{\max }
$$

where $P_{T}=P_{1}+P_{2}+P_{r}$, and $P_{r}$ is the sum of transmit power of CR nodes and $P_{T}^{\max }$ is the maximum transmit power of the total network. Note that $P_{1}$ and $P_{2}$ are assumed constant in this problem because generally, the secondary network is not allowed to control the power of the primary transmitter, and here our optimization problem is solved at the secondary network.

We define $\mathbf{X}=\mathbf{w} \mathbf{w}^{H}$ and note that $\mathbf{X}=\mathbf{w} \mathbf{w}^{H}$ is equivalent to $\mathbf{X}$ which is a rank one symmetric positive semidefinite (PSD) matrix. We change the form of the problem as:

$$
\begin{aligned}
\max _{\mathbf{X}} & \frac{\operatorname{Tr}\left[\left(P_{1} \mathbf{H}^{\prime}+P_{2} \mathbf{H}^{\prime \prime}\right) \mathbf{X}\right]}{\sigma^{2}+\operatorname{Tr}\left[\sigma^{2} \mathbf{D}_{3} \mathbf{X}\right]} \\
\text { s.t. } & \operatorname{Tr}\left(\left(P_{2} \mathbf{H}-\gamma_{1} \mathbf{D}_{1}\right) \mathbf{X}\right) \geq \gamma_{1}, \operatorname{Tr}\left(\left(P_{1} \mathbf{H}-\gamma_{2} \mathbf{D}_{2}\right) \mathbf{X}\right) \geq \gamma_{2}, \\
& P_{1}+P_{2}+\operatorname{Tr}\left[\left(P_{1} \mathbf{D}_{1}+P_{2} \mathbf{D}_{2}+\sigma^{2}\right) \mathbf{X}\right] \leq P_{T}^{\max }, \\
& \mathbf{X}=\mathbf{w w}^{H}, \mathbf{X} \succeq 0 \text { and } \operatorname{rank}(\mathbf{X})=1,
\end{aligned}
$$

where $\mathbf{w}^{H} \mathbf{A} \mathbf{w}=\operatorname{Tr}\left[\mathbf{A}\left(\mathbf{w} \mathbf{w}^{H}\right)\right]$, and $\mathbf{H}=\mathbf{h h}^{H}, \mathbf{H}^{\prime}=\mathbf{h}^{\prime} \mathbf{h}^{\prime H}$ and $\mathbf{H}^{\prime \prime}=$ $\mathbf{h}^{\prime \prime} \mathbf{h}^{\prime \prime H}$. By using the idea of semidefinite relaxation and dropping the nonconvex rank-one constraint, the problem can be relaxed as:

$$
\begin{array}{rl}
\max _{\mathbf{X}, t} & t \\
\text { s.t. } & \operatorname{Tr}\left[\left(P_{1} \mathbf{H}^{\prime}+P_{2} \mathbf{H}^{\prime \prime}-t \sigma^{2} \mathbf{D}_{3}\right) \mathbf{X}\right] \geq \sigma^{2} t \\
& \operatorname{Tr}\left(\left(P_{2} \mathbf{H}-\gamma_{1} \mathbf{D}_{1}\right) \mathbf{X}\right) \geq \gamma_{1}, \operatorname{Tr}\left(\left(P_{1} \mathbf{H}-\gamma_{2} \mathbf{D}_{2}\right) \mathbf{X}\right) \geq \gamma_{2},
\end{array}
$$




$$
P_{1}+P_{2}+\operatorname{Tr}\left[\left(P_{1} \mathbf{D}_{1}+P_{2} \mathbf{D}_{2}+\sigma^{2}\right) \mathbf{X}\right] \leq P_{T}^{\max }, \text { and } \mathbf{X} \succeq 0 .
$$

In (9), if the value of $t$ is kept fixed, the set of feasible $\mathbf{X}$ is convex. By solving (9), the maximum achievable SNR at the CBS can be resulted in which the maximum value of $t$, denoted as $t_{\max }$. To solve (9), we use an iterative algorithm provided in [2]. For some given SNR value $t$, the convex feasibility problem:

find $\mathbf{X}$

$$
\begin{array}{ll}
\text { s.t. } & \operatorname{Tr}\left[\left(P_{1} \mathbf{H}^{\prime}+P_{2} \mathbf{H}^{\prime \prime}-t \sigma^{2} \mathbf{D}_{3}\right) \mathbf{X}\right] \geq \sigma^{2} t \\
& \operatorname{Tr}\left(\left(P_{2} \mathbf{H}-\gamma_{1} \mathbf{D}_{1}\right) \mathbf{X}\right) \geq \gamma_{1}, \operatorname{Tr}\left(\left(P_{1} \mathbf{H}-\gamma_{2} \mathbf{D}_{2}\right) \mathbf{X}\right) \geq \gamma_{2}, \\
& P_{1}+P_{2}+\operatorname{Tr}\left[\left(P_{1} \mathbf{D}_{1}+P_{2} \mathbf{D}_{2}+\sigma^{2}\right) \mathbf{X}\right] \leq P_{T}^{\max } \text { and } \mathbf{X} \succeq 0 .
\end{array}
$$

is feasible, then $t_{\max } \geq t$ and if (10) is not feasible, then $t_{\max }<t$. Based on this observation, one can check whether the optimal value $t_{\max }$ of the quasi-convex problem (9) is smaller or greater than any given value of $t$. To find the maximum value of $t$ while holding the feasibility of problem, we can use a simple bisection algorithm provided in Table. I.

Table I. Iterative algorithm for finding the optimal point $t$.

\begin{tabular}{ll}
\hline Step 1 & Properly set the initial values of $t_{l}$ and $t_{u}$. \\
\hline Step 2 & Set $t:=\left(t_{l}+t_{u}\right) / 2$ and solve $(10)$. \\
\hline Step 3 & If $(10)$ is feasible, then $t_{l}:=t ;$ otherwise $t_{u}:=t$. \\
\hline Step 4 & If $t_{u}-t_{l}<\delta$, then go to Step $5 ;$ otherwise go to Step 2. \\
\hline Step 5 & $\begin{array}{l}\text { Find the weight vector from the principal eigenvector } \\
\text { of the resulting matrix } X_{\text {opt }} .\end{array}$ \\
\hline
\end{tabular}

Let us start with some preselected interval $\left[\begin{array}{ll}t_{l} & t_{u}\end{array}\right]$ that is known to contain the optimal value $t_{\max }$, where the problem (10) is solved at the midpoint $t=\left(t_{l}+t_{u}\right) / 2$. If (10) is feasible for this value of $t$, then $t_{l}=t$ is set; otherwise $t_{u}=t$ is chosen. This procedure is repeated until the difference between $t_{u}$ and $t_{l}$ is smaller than some preselected threshold.

\section{Numerical results}

We assume that the transmit power of two transceivers are both equal to $10 \mathrm{dBW}$ (i.e., $P_{1}=P_{2}=10 \mathrm{dBW}$ ). The optimization problem is solved by using the convex optimization toolbox CVX [3]. For performance comparison, we consider two competitive beamforming techniques. We first consider an uniform beamforming weighting vector for relay nodes in which, to ensure a fair comparison, the total transmit power of relays is equal to the power achieved by our proposed method, but this power is uniformly distributed among relays. Then we consider a conventional beamforming, i.e., without any cognitive radio capabilities where the weighting vector is set so as to minimize the total power dissipated in the two-way relay network.

This comparison is shown in Fig. 2 (a), where we compare the receiver operating characteristic (ROC) of our proposed beamforming method with 


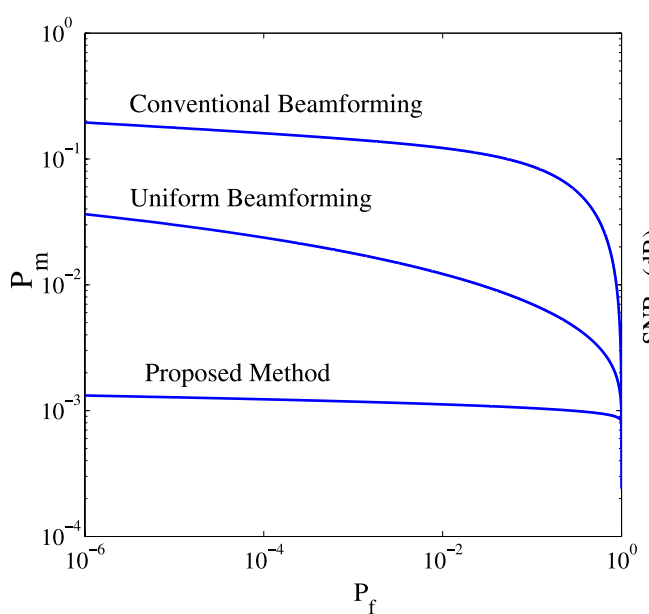

(a)

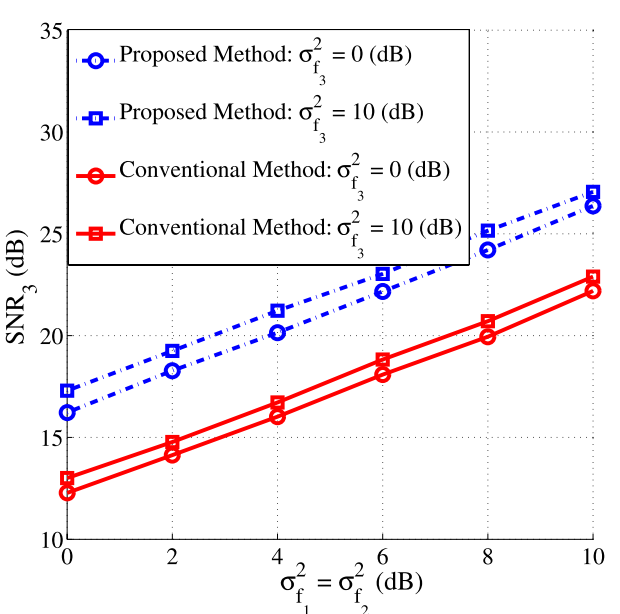

(b)

Fig. 2. (a) Probability of miss-detection versus the probability of false-alarm (ROC) for proposed distributed beamforming, uniform beamforming and conventional beamforming; $n_{r}=10$ and $\gamma_{1}=$ $\gamma_{2}=10 \mathrm{~dB}$. (b) The performance of spectrum sensing versus the channel variances $\sigma_{f_{1}}^{2}=\sigma_{f_{2}}^{2}$ for different channel variances $\sigma_{f_{3}}^{2} ; n_{r}=10$ and $\gamma_{1}=\gamma_{2}=10 \mathrm{~dB}$.

ROCs obtained with uniform beamforming and the conventional method. This figure illustrates that considering the proposed method reduces significantly the miss-detection probability while providing the requirements at the primary network, compared to uniform weight beamforming and conventional methods. The effect of channel variations in the performance of spectrum sensing in terms of the SNR at the CBS is depicted in Fig. 2 (b). This figure depicts the SNR at the CBS versus the channel variances $\sigma_{f_{1}}^{2}$ and $\sigma_{f_{2}}^{2}$ for different achieved by using the proposed and the conventional beamforming methods, respectively. It is clearly seen from this figure that the proposed method increases the SNR at the CBS compared to the conventional method. Thus our proposed technique increases the SNR and thus the accuracy of spectrum sensing at the CBS.

\section{Conclusion}

In this work, we investigated the problem of distributed beamforming in a two-step two-way cognitive radio network. For a given set of minimum required SNR at the transceivers, an optimal beamforming weight vector was obtained to maximize the SNR at the CBS, with the aim of increasing the accuracy of spectrum sensing. The optimization problem was relaxed to semidefinite form and solved efficiently by an iterative algorithm. Simulation results demonstrated that our proposed SNR maximization method improves the performance of spectrum sensing in comparison with uniform and conventional two-way relay beamforming methods. 